\title{
21 Chronische Obstipation
}

Katrin Heilmann, Christian Gingert, Franc Hetzer

\subsection{Einleitung}

Obstipation ist nicht als eigenständiges Krankheitsbild anzusehen, sondern vielmehr als ein Symptom anderer Ursachen. Man unterscheidet einfache von schwerwiegenden Formen, außerdem gilt es die akute von der chronischen Obstipation abzugrenzen. Der Leidensdruck der Patienten ist v. a. bei der chronischen Obstipation häufig hoch und die Therapieoptionen vielfältig.

Die Motilitätsstörung, die der Slow-Transit-Obstipation zugrunde liegt, kann häufig konservativ behandelt werden.

Die Outlet-Obstipation ist entweder auf ein strukturelles Problem oder auch funktionelle Auslöser im Bereich des Beckenbodens zurückzuführen. Letztere sollten primär konservativ und ergänzend mit einer Beckenbodentherapie therapiert werden. Biofeedbackübungen sind vielversprechend bei Dyssynergie; bei Sensibilitätsstörungen kommen Sensibilisierungsübungen zum Einsatz zur Reaktivierung des Defäkationsreizes (vgl. Kap. 13 Stuhlentleerungstörung, Band 1).

Primäres Ziel vor Einleitung einer Therapie ist stets die Ursachenforschung: Welche Ursachen liegen der Obstipation im individuellen Fall zugrunde? Dabei gilt es zu berücksichtigen, dass die Darmmotilität und die Defäkation auf einem komplexen Zusammenspiel zwischen zentralem und autonomem Nervensystem sowie dem Gastrointestinaltrakt bis zum Beckenboden und Sphinkterapparat beruht. Um langfristig Erfolg in der Behandlung der chronischen Obstipation zu haben, ist außerdem - wie so oft - das Vertrauensverhältnis des Patienten zum behandelnden Arzt von entscheidender Bedeutung.

\subsection{Definition und Klassifikation}

\subsubsection{Akute Obstipation}

Die akute Obstipation umschreibt eine plötzlich auftretende Stuhlentleerungsstörung. Meist geht dies mit einer Schmerzproblematik einher und die häufigsten Ursachen für eine akute Obstipation sind entzündliche (wie bei der Divertikulitis) oder auch karzinombedingte Stenosen. Typischerweise führt der Defäkationsschmerz zu einem Beckenbodenspasmus, der eine Defäkation verhindert. Eine rasche Abklärung ist beim älteren Patienten anzustreben. 


\subsubsection{Chronische Obstipation}

Auf internationaler Ebene wurden Definitionen erstellt, die sowohl subjektive Beschwerden des Patienten (z. B. starkes Pressen) wie auch objektive Parameter (z. B. Stuhlfrequenz) berücksichtigen. Bewährt haben sich hier die Rom-IV-Kriterien:

Gemäß den Rom-IV-Kriterien liegt eine chronische Obstipation vor, wenn in den letzten 6 Monaten unbefriedigende Stuhlentleerungen über mindestens 3 Monate bestanden und zumindest 2 der folgenden Symptome zusätzlich vorliegen:

- Pressen beim Stuhlgang in mehr als $25 \%$ der Fälle

- Gefühl der inkompletten Entleerung nach dem Stuhlgang in mehr als 25\% der Fälle

- harter oder knollenartiger Stuhl in mehr als 25\% der Fälle

- manuelle Manöver zur Erleichterung der Stuhlentleerung in mehr als 25\% der Fälle

- Stuhlfrequenz $<3$ pro Woche

In der weiteren Klassifikation unterscheidet man die primäre Obstipation von sekundären Formen. Während sich die primäre Form auf die Darmfunktion bezieht, liegen der sekundären Form extrakolonische Ursachen zugrunde. Die primäre Obstipation beruht auf einem zu langsamen Transport im Darm (Slow-Transit-Obstipation) oder einer Stuhlentleerungsstörung (Outlet-Obstipation), wobei auch Mischformen auftreten können (Abb. 21.1).

Die obstipative Variante des Reizdarm-Syndroms (Constipation-predominant Irritable Bowel Syndrome, IBS.C) ist als Differentialdiagnose anzusehen und wird nicht zur Obstipation im engeren Sinn gezählt.

Die Ätiologie der sekundären Obstipation ist mannigfaltig, jedoch spielen Medikamente bzw. deren Nebenwirkungen im klinischen Alltag zweifellos eine große Rolle (Tab. 21.1).

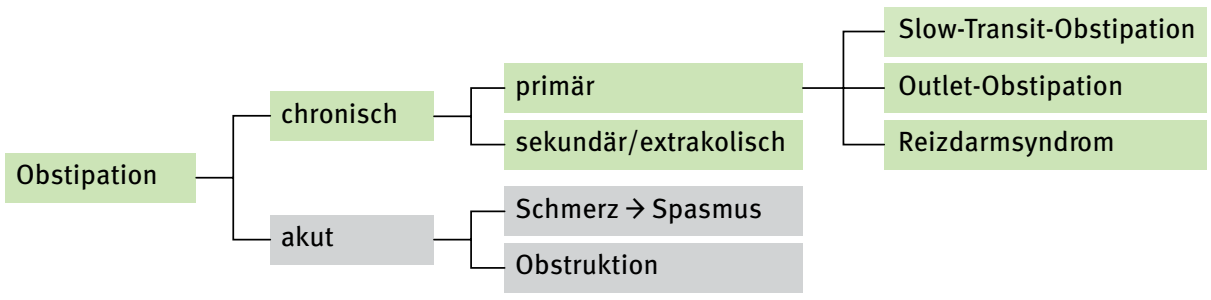

Abb. 21.1: Einteilung der Obstipation nach Grundformen und Ursachen. 
Tab. 21.1: Extrakolonische Gründe für die Obstipation.

\begin{tabular}{|c|c|c|c|c|c|}
\hline $\begin{array}{l}\text { endokrin und } \\
\text { metabolisch }\end{array}$ & $\begin{array}{l}\text { neurologisch } \\
\text { zerebral }\end{array}$ & & peripher & Medikamente & Myopathie \\
\hline $\begin{array}{l}\text { - Diabetes } \\
\text { mellitus } \\
\text { - Glykagonom } \\
\text { - Hyperkalzä- } \\
\text { mie } \\
\text { - Hyperparathy- } \\
\text { reoidismus } \\
\text { - Hypokaliämie } \\
\text { - Hypopituita- } \\
\text { rismus } \\
\text { - Hypothyreose } \\
\text { - Milch-Alkali- } \\
\text { Syndrom } \\
\text { - Phäochromo- } \\
\text { zytom } \\
\text { - Porphyrie } \\
\text { - Schwanger- } \\
\text { schaft } \\
\text { - Urämie }\end{array}$ & $\begin{array}{l}\text { - Morbus } \\
\text { Parkinson } \\
\text { - Schlag- } \\
\text { anfall } \\
\text { - Tumor }\end{array}$ & $\begin{array}{l}\text { - Cauda-equi- } \\
\text { na-Tumor } \\
\text { - Ischämie } \\
\text { - iatrogen } \\
\text { - Meningo- } \\
\text { zele } \\
\text { - Multiple } \\
\text { Sklerose } \\
\text { - Paraplegie } \\
\text { - Shy-Drager- } \\
\text { Syndrom } \\
\text { - Tabes } \\
\text { dorsalis } \\
\text { - Trauma }\end{array}$ & $\begin{array}{l}\text { - } \text { autonome } \\
\text { Neuropathie } \\
\text { - Chagas- } \\
\text { Krankheit } \\
\text { - MEN-Syn- } \\
\text { drom } \\
\text { - Morbus } \\
\text { Reckling- } \\
\text { hausen }\end{array}$ & $\begin{array}{l}\text { - Anästhetika } \\
\text { - Analgetika } \\
\text { - Antazida } \\
\text { (Kalzium und } \\
\text { Aluminium) } \\
\text { - Anticholiner- } \\
\text { gika } \\
\text { - Antikonvul- } \\
\text { siva } \\
\text { - Antidepres- } \\
\text { siva } \\
\text { - Anti-Parkin- } \\
\text { son- Mittel } \\
\text { - Bariumsulfat } \\
\text { - Kalziumago- } \\
\text { nisten } \\
\text { - Diuretika } \\
\text { - Ganglien- } \\
\text { blocker } \\
\text { - Hypotensiva } \\
\text { - MAO-Inhibi- } \\
\text { toren } \\
\text { - Metalle } \\
\text { (Arsen, Phos- } \\
\text { phor, Queck- } \\
\text { silber) } \\
\text { - Opiate } \\
\text { - Psychothera- } \\
\text { peutika } \\
\text { - }\end{array}$ & $\begin{array}{l}\text { - Amyloidose } \\
\text { - Dermato- } \\
\text { myositis } \\
\text { - myotone } \\
\text { Dystrophie } \\
\text { - Skleroder- } \\
\text { mie }\end{array}$ \\
\hline
\end{tabular}

\subsubsection{Slow-Transit-Obstipation}

Bei dieser Form der chronischen Obstipation handelt es sich um einen zu langsamen Transport im Kolon bzw. eine verlängerte Kolontransitzeit. Eine intakte sensomotorische Funktion des Darms ist Voraussetzung für eine regelrechte intestinale Motilität und wird maßgeblich durch das enterische Nervensystem, die glatte Darmwandmuskulatur sowie die intestinalen Schrittmacherzellen (interstitielle Cajal-Zellen, ICC) vermittelt. Isolierte oder kombinierte Schädigungen dieser Strukturen können zu intestinalen Motilitätsstörungen führen und werden als gastrointestinale neuromuskuläre Pathologien (GINMP) bezeichnet. In der «Londoner Klassifikation» werden 
sie in folgende Formen unterteilt: (1) enterische Neuropathien, (2) enterische Myopathien, (3) Veränderungen der ICC. GINMP sind in der Regel nur bei schweren Formen der Obstipation nachweisbar und zeigen unterschiedliche Ausprägungen. Die histopathologischen Veränderungen sollten nicht zwingend als kausale, sondern als assoziierte morphologische Korrelate einer Obstipation bewertet werden. Es wird angenommen, dass die GINMP bei Patienten mit schwerer Obstipation (Slow-transitObstipation) zu folgenden pathophysiologischen Veränderungen führen und damit eine Verlängerung der Kolontransitzeit bedingen: (1) Verminderte Sensitivität und Compliance des Rektums, (2) Erhöhung retrograder und Verminderung anterograder Kontraktionswellen im Kolon, (3) Verringerung der Kontraktionsstärke und -frequenz im Kolon. Bei Patienten mit chronischer Obstipation wurden folgende Formen einer enterischen Neuropathie beschrieben: Hypoganglionose des Plexus myentericus, degenerative Neuropathie, intestinale neuronale Dysplasie, enterische Ganglionitis, veränderter Neurotransmitterstatus. Größte Übereinstimmung laut Studienlage stellt die Hypoganglionose des Plexus myentericus bei Slow-Transit-Obstipation dar. Bei Patienten mit chronischer Obstipation wurden folgende Formen einer enterischen Myopathie beschrieben: degenerative Fibrosierung, enterische Leiomyositis, amphophile Einschlusskörperchen, Myofilament-Verlust, atrophe Desmosis coli. Nach Studienlage stellt jedoch keiner dieser histopathologischen Phänotypen einen konsistenten Befund dar. Bei Patienten mit Slow-Transit-Obstipation wurde mehrheitlich eine signifikante Abnahme der ICC ( $<50 \%$ der Norm) beobachtet. Allerdings bleibt unklar, ob der Verlust von ICC ursächlich zur Entwicklung einer chronischen Obstipation beiträgt oder lediglich Folge der verlangsamten Darmpassage ist. Zusammenfassend ist die Slow-Transit-Obstipation eine Dysfunktion der sensomotorischen Darmfunktion des enterischen Nervensystems und der interstitiellen Cajal-Zellen (Schrittmacherzellen) sowie der glatten Darmwandmuskulatur. Diese Transportstörung kann in ihrer Maximalvariante den gesamten Gastrointestinaltrakt betreffen. In der Literatur werden auch Obstipationsformen wie die angeborene oder erworbene Aganglionose M. Hirschsprung bzw. der M. Chagas, die intestinale neuronale Dysplasie, die Dysganglionose, die intestinale Myopathie und das Megakolon bzw. -rektum subsummiert. Obstipation kann mit Veränderungen an intestinalen Schrittmacherzellen (interstitielle Cajal-Zellen) sowie mit enterischen Neuropathien und/oder Myopathien assoziiert sein.

Von der Slow-Transit-Obstipation sind nahezu ausschliesslich Frauen betroffen, wobei die Symptome meist in der Pubertät anfangen. Typischerweise berichten die Patientinnen über eine Stuhlentleerungsfrequenz von weniger als 2-mal pro Woche. 


\subsubsection{Outlet-Obstipation}

Die Stuhlentleerungsstörung oder Outlet-Obstruktion hat vielfältige Ursachen und ist entweder struktureller Natur, d.h. anatomisch-bedingt, oder auf funktionelle Störungen zurückzuführen. $\mathrm{Zu}$ den strukturellen Störungen werden die Prolapserkrankungen gezählt. Man grenzt hier den inneren Vorfall (die Intussuszeption) von dem nach außen tretenden Rektumprolaps ab. Was in der Theorie banal imponiert, erweist sich in der Praxis für Nichtproktologen häufig als Herausforderung, da die Intussuszeption meist nicht einfach zu sehen ist und als Symptom die Inkontinenz im Vordergrund stehen kann, wenn es beim manifesten Rektumprolaps zu einer starken Überdehnung des Beckenbodens und Sphinkterapparates kommt (vgl. Kapitel 13, Stuhlentleerungsstörung, Band 1).

Eine schwere chronifizierte Outlet-Obstipation kann sekundär die Kolonmotilität negativ beeinflussen. Dieses Mischbild der Motilitäts- und Entleerungsstörung ist nicht selten und muss erkannt werden, um eine sinnvolle Therapie etablieren zu können. In der Regel erholt sich die Transitverzögerung nach Korrektur der OutletObstruktion. Dies dauert meist einige Wochen und Monate und kann durch prokinetische Darmstimulantien unterstützt werden (z. B. Bisacodyl, Natriumpicosulfat, Senna alexandrina). Tab. 21.2 gibt einen Überblick über die Krankheitsbilder der Entleerungsstörungen.

Tab. 21.2: Krankheitsbilder unter dem Sammelbegriff „Entleerungsstörungen“ (BAS, ODS) (nach A. Herold).

\begin{tabular}{|c|c|c|c|}
\hline & $\begin{array}{l}\text { funktionelle } \\
\text { Störungen }\end{array}$ & morphologisch-organische Störungen & $\begin{array}{l}\text { Kombinationen aus } \\
\text { funktionellen und mor- } \\
\text { phologisch-organischen } \\
\text { Störungen }\end{array}$ \\
\hline $\begin{array}{l}\text { Becken- } \\
\text { boden }\end{array}$ & $\begin{aligned} & \text { - Fehlkoordina- } \text { tion Anismus } \\
& \text { - Spastik } \\
& \text { - psychogene } \\
& \text { Ursachen }\end{aligned}$ & $\begin{array}{l}\text { - Myopathie des M. internus } \\
\text { - Internushypertrophie } \\
\text { - dorsale Sphinkterdysplasie } \\
\text { - anorektale Stenose } \\
\text { - Analfissur }\end{array}$ & $\begin{array}{l}\text { keine eigene Syndrome, } \\
\text { die Kombi ist absolut frei } \\
\text { zusammengesetzt }\end{array}$ \\
\hline $\begin{array}{l}\text { Kolon und } \\
\text { Rektum }\end{array}$ & $\begin{array}{l}\text { - idiopathische } \\
\text { Inertia recti } \\
\text { - gestörte } \\
\text { autonome In- } \\
\text { nervation }\end{array}$ & $\begin{array}{l}\text { - postoperative Inertia recti } \\
\text { - Dysganglionose } \\
\text { - mangelhafte Internusrelaxation } \\
\text { - Morbus Hirschsprung } \\
\text { - Enterozele } \\
\text { - Rektozele } \\
\text { - Rektumprolaps } \\
\text { - reduzierte Rektum-Compliance } \\
\text { - obstruierender Tumor }\end{array}$ & $\begin{array}{l}\text { keine eigene Syndrome, } \\
\text { die Kombi ist absolut frei } \\
\text { zusammengesetzt }\end{array}$ \\
\hline
\end{tabular}




\subsection{Epidemiologie}

Zur Prävalenz der Obstipation finden sich je nach Region und Population unterschiedliche Angaben. Im europäischen Durchschnitt liegt die Prävalenz bei 15-19,2 \% mit einer deutlichen Zunahme bei über 65-Jährigen, wobei das weibliche Geschlecht insgesamt signifikant häufiger von diesem Symptom betroffen ist (21 vs. $8 \%$ ). Patienten mit chronischer Obstipation verursachen im Vergleich zur übrigen nichtobstipierten Bevölkerung höhere direkte Kosten (durch Verschreibungen, Arztkonsultationen, Hospitalisationen) wie auch indirekte Kosten (durch Arbeitsunfähigkeit).

\subsection{Diagnostik}

Um die Diagnose der chronischen Obstipation im klinischen Alltag stellen zu können, müssen die Kriterien gemäss der vorher erwähnten Rom-IV-Klassifikation erfüllt sein. In der weiteren Differenzierung müssen für die Diagnose einer Slow-Transit-Obstipation eine verlängerte Kolontransitzeit vorliegen und bei der Outlet-Obstruktion zusätzliche Defäktionstests ein pathologisches Resultat aufweisen.

\subsection{Anamnese}

In der Gesellschaft handelt es sich bei der Defäkation nach wie vor um ein tabuisiertes Thema. Umso entscheidender ist eine gute und empathische Anamnese, die zunächst allgemeine Angaben zur Vorgeschichte (Ernährungsgewohnheiten, beruf-

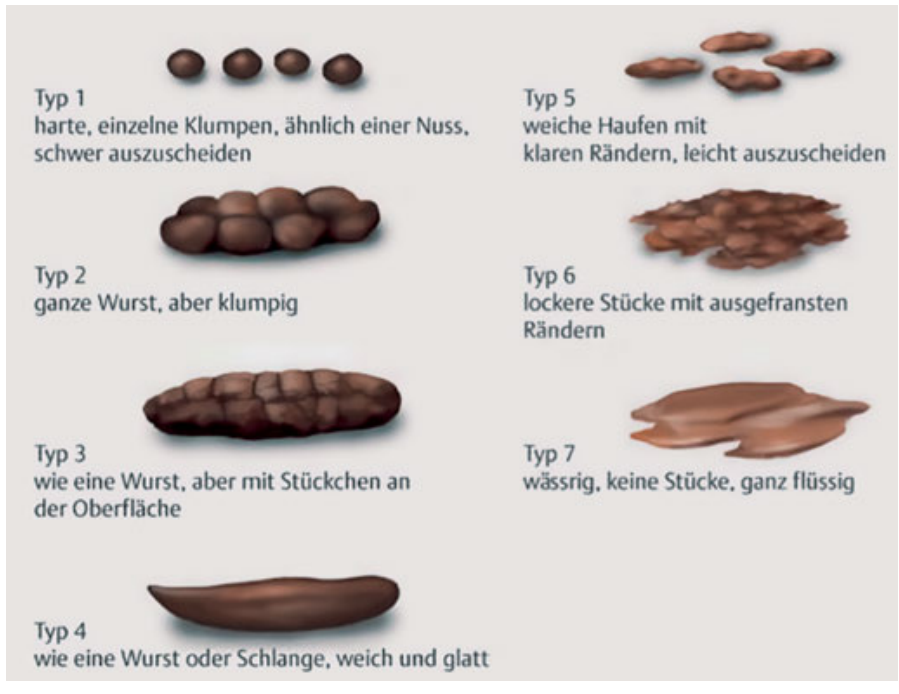

Abb. 21.2: BristolStuhlformen-Skala. (Quelle: Füeßl, Middeke: Duale Reihe Anamnese und Klinische Untersuchung, 4. Auflage 2000, Georg Thieme Verlag, Stuttgart. Mit freundlicher Genehmigung des Thieme Verlags). 
liche Tätigkeit, Geburten, abdominelle Voroperationen, Medikamente u. a.) erfasst. Anschliessend sollten Stuhlgewohnheiten erfragt werden. Hier gilt es, Stuhlfrequenz, Stuhlkonsistenz, Entleerungsablauf, Stuhldrang u. a. zu ermitteln. Hilfestellung bei einer möglichst standardisierten Anamnese bietet die Bristol-Stool-Skala (Abb. 21.2).

Zusätzlich kommen validierte Fragebögen zum Einsatz, die der Patient im Idealfall bereits vor der Konsultation erhält, um sich so auf die Konsultation vorzubereiten und die Symptome genauer zu reflektieren.

Beispielhaft sind hier für die Slow-Transit-Obstipation der Cleveland Constipation Score (Tab. 21.3) und für die Outlet-Obstipation der Obstructed Defecation Syndrome (ODS) Longo Score (Tab. 21. 4).

Tab. 21.3: Cleveland Clinic Obstipation Score.

\begin{tabular}{|c|c|c|c|c|c|c|}
\hline Frage & 0 & 1 & 2 & 3 & 4 & $\begin{array}{l}\text { Punkte } \\
\text { gesamt }\end{array}$ \\
\hline $\begin{array}{l}\text { Wie oft haben Sie Stuhl- } \\
\text { gang? }\end{array}$ & $\begin{array}{l}\text { fast täg- } \\
\text { lich }\end{array}$ & $\begin{array}{l}2 \times / \\
\text { Woche }\end{array}$ & $\begin{array}{l}1 \times / \text { Wo- } \\
\text { che }\end{array}$ & $\begin{array}{l}\text { seltener als } \\
1 \times / \text { Woche }\end{array}$ & $\begin{array}{l}\text { seltener als } \\
1 \times / \text { Monat }\end{array}$ & \\
\hline $\begin{array}{l}\text { Wie oft ist die Stuhlentlee- } \\
\text { rung schwierig und/oder } \\
\text { schmerzhaft? }\end{array}$ & nie & selten & $\begin{array}{l}\text { gelegent- } \\
\text { lich }\end{array}$ & meistens & immer & \\
\hline $\begin{array}{l}\text { Wie häufig haben Sie das } \\
\text { Gefühl der unvollständigen } \\
\text { Entleerung? }\end{array}$ & nie & selten & $\begin{array}{l}\text { gelegent- } \\
\text { lich }\end{array}$ & meistens & immer & \\
\hline $\begin{array}{l}\text { Wie häufig haben Sie Bauch- } \\
\text { schmerzen? }\end{array}$ & nie & selten & $\begin{array}{l}\text { gelegent- } \\
\text { lich }\end{array}$ & meistens & immer & \\
\hline $\begin{array}{l}\text { Wie viele Minuten verbrin- } \\
\text { gen Sie pro Entleerungsver- } \\
\text { such auf der Toilette? }\end{array}$ & $<5$ & $5-10$ & $10-20$ & $20-30$ & $>30$ & \\
\hline $\begin{array}{l}\text { Unterstützende Maßnah- } \\
\text { men? }\end{array}$ & keine & $\begin{array}{l}\text { Abführ- } \\
\text { mittel }\end{array}$ & $\begin{array}{l}\text { Einläufe } \\
\text { (manuelle } \\
\text { Entlee- } \\
\text { rung) }\end{array}$ & & & \\
\hline $\begin{array}{l}\text { Wie häufig sind erfolglose } \\
\text { Versuche der Darmentlee- } \\
\text { rung pro } 24 \text { Stunden? }\end{array}$ & 0 & $1-3$ & $3-6$ & $6-9$ & $>9$ & \\
\hline $\begin{array}{l}\text { Wie lange leiden Sie schon } \\
\text { an Verstopfung (Jahre)? }\end{array}$ & 0 & $1-5$ & $5-10$ & $10-20$ & $>20$ & \\
\hline $\begin{array}{l}\text { Gesamtscore: maximal } 30 \text {; } \\
\text { Verstopfung > } 15 \text { Punkte }\end{array}$ & & & & & & \\
\hline
\end{tabular}


Tab. 21.4: Longo ODS-Score: 0-40 Punkte.

\begin{tabular}{|c|c|c|c|c|c|c|c|c|c|}
\hline \multicolumn{10}{|l|}{ Frage } \\
\hline $\begin{array}{l}\text { Defäkations- } \\
\text { frequenz (Ant- } \\
\text { wort, Punkte) }\end{array}$ & $\begin{array}{l}1-2 \times / \\
1-2 \text { Tage }\end{array}$ & 0 & $\begin{array}{l}2 \times / \text { Woche } \\
\text { oder } 3 \text { Ver- } \\
\text { suche/Tag }\end{array}$ & 1 & $\begin{array}{l}1 \times / \text { Woche } \\
\text { oder } 4 \text { Ver- } \\
\text { suche/Tag }\end{array}$ & 2 & $\begin{array}{l}<1 \times / \text { Woche } 0 \\
\text { suche } / \text { Tag }\end{array}$ & der 4 Ver- & 3 \\
\hline \multicolumn{10}{|c|}{ Pressen beim Stuhlgang } \\
\hline Intensität & $\begin{array}{l}\text { keine, } \\
\text { leicht }\end{array}$ & 0 & moderat & 1 & intensiv & 2 & & & \\
\hline Dauer & & & kurzfristig & 1 & verlängert & 2 & & & \\
\hline $\begin{array}{l}\text { Gefühl der } \\
\text { inkompletten } \\
\text { Entleerung }\end{array}$ & niemals & 0 & $\leq 1 \times$ /Woche & 1 & $2 \times /$ Woche & 2 & $>2 \times /$ Woche & & 3 \\
\hline $\begin{array}{l}\text { rektale/ } \\
\text { perineale Be- } \\
\text { schwerden/ } \\
\text { Schmerzen }\end{array}$ & niemals & 0 & $\leq 1 \times /$ Woche & 1 & $2 \times /$ Woche & 2 & $>2 \times /$ Woche & & 3 \\
\hline $\begin{array}{l}\text { Aktivitätsein- } \\
\text { schränkung/ } \\
\text { Woche }\end{array}$ & keine & 0 & $<25 \%$ & 2 & $25-50 \%$ & 4 & $>50 \%$ & & 6 \\
\hline Laxanzien & keine & 0 & $\begin{array}{l}<25 \% \text { der } \\
\text { Defäkationen }\end{array}$ & 1 & $\begin{array}{l}25-50 \% \text { der } \\
\text { Defäkationen }\end{array}$ & 3 & $\begin{array}{l}>50 \% \text { der } \\
\text { Defäkationen }\end{array}$ & 5 immer & 7 \\
\hline Einläufe & keine & 0 & $\begin{array}{l}<25 \% \text { der } \\
\text { Defäkationen }\end{array}$ & 1 & $\begin{array}{l}25-50 \% \text { der } \\
\text { Defäkationen }\end{array}$ & 3 & $\begin{array}{l}>50 \% \text { der } \\
\text { Defäkationen }\end{array}$ & 5 immer & 7 \\
\hline Digitation & keine & 0 & $\begin{array}{l}<25 \% \text { der } \\
\text { Defäkationen }\end{array}$ & 1 & $\begin{array}{l}25-50 \% \text { der } \\
\text { Defäkationen }\end{array}$ & 3 & $\begin{array}{l}>50 \% \text { der } \\
\text { Defäkationen }\end{array}$ & 5 immer & 7 \\
\hline
\end{tabular}

Gesamtscore: maximal 40 Punkte; kein „cut-off“-Wert publiziert

\subsection{Proktologische Untersuchung}

Die Inspektion und digitale Untersuchung liefern v. a. bei Patienten mit akuter Obstipation oft wertvolle Hinweise auf deren Ätiologie. So lassen sich hier beispielweise Diagnosen wie Analfissur oder Hämorrhoidalthrombose ohne weiterführende Abklärungen bereits durch sorgfältige Inspektion und digitale Untersuchung stellen. Bei der chronischen Obstipation auf dem Boden einer Transportstörung ist die klinische Untersuchung meist weniger richtungsweisend. Jedoch ist sie zweifelsohne obligat, um einen allfälligen Prolaps zu diagnostizieren oder die Beckenbodensynergie zu beurteilen, indem überprüft wird, ob der Patient die Kommandos „Pressen“ und „Kneifen“ richtig umsetzen kann. 
Mittels Proktoskopie erfolgt die Beurteilung des Analkanals. Pathologische Befunde wie Hämorrhoidalknoten oder Analfissuren lassen sich mit dieser Untersuchung gut feststellen.

Weitere organische Ursachen werden in der Rektoskopie gesucht bzw. ausgeschlossen: Tumoren im Mastdarm, Intussuszeptionen (innere Vorfälle) und nach außen tretende Prolapse werden direkt diagnostiziert bzw. gibt es mitunter indirekte Hinweise darauf in Form von Schleimhautveränderungen wie Ulzerationen.

\subsection{Weiterführende Diagnostik}

Bei Patienten, die auch nach konservativen Massnahmen bzw. Lifestyle-Veränderungen (Ernährungsumstellung, Stuhlregulation u. a.) unter persistierenden Symptomen leiden, stehen diverse Methoden der apparativen Diagnostik zur Verfügung.

Je nachdem, ob eher der Verdacht auf eine Slow-Transit-Obstipation oder eine Outlet-Symptomatik besteht, stehen am Anfang der weiterführenden Diagnostik die Messung der Kolontransitzeit (bei V. a. Slow-Transit) oder die Defäkografie (bei V.a. Outlet-Obstipation).

\subsubsection{Kolontransitzeit}

Die Kolontransitzeit KTZ wird in der Regel mit röntgendichten Markern gemessen. Andere Verfahren basieren auf szintigrafischen Messungen mit Technetium-99m oder Indium-111. Im Hinton-Test schluckt der Patient eine Kapsel mit 10 Opaque-Markern an 6 aufeinanderfolgenden Tagen. An Tag 7 erfolgt eine Abdomenleeraufnahme, in der die die im Kolon verbliebenen Marker gezählt werden. Die Kolontransitzeit (KTZ) ergibt sich aus Summe der Marker $\times 2,4$. Die durchschnittliche KTZ beträgt 30-40 Stunden. Bei der Auswertung können auch rechtes Hemikolon $(11,3 \pm 1,1 \mathrm{~h})$ und Rektosigmoid $(12,4 \pm 1,1 \mathrm{~h})$ für sich einzeln betrachtet werden. Als Grenzwerte der gesamten Kolontransitzeit gelten für Frauen ca. 70 h, für Männer ca. 60 h. Eine Kolontransitzeit über $72 \mathrm{~h}$ ist per definitionem als Slow-Transit-Obstipation anzusehen (vgl. Abb.21.3 und 21.4). Voraussetzung für verwertbare Ergebnisse ist der komplette Verzicht auf Laxanzien während der Untersuchungsdauer. 


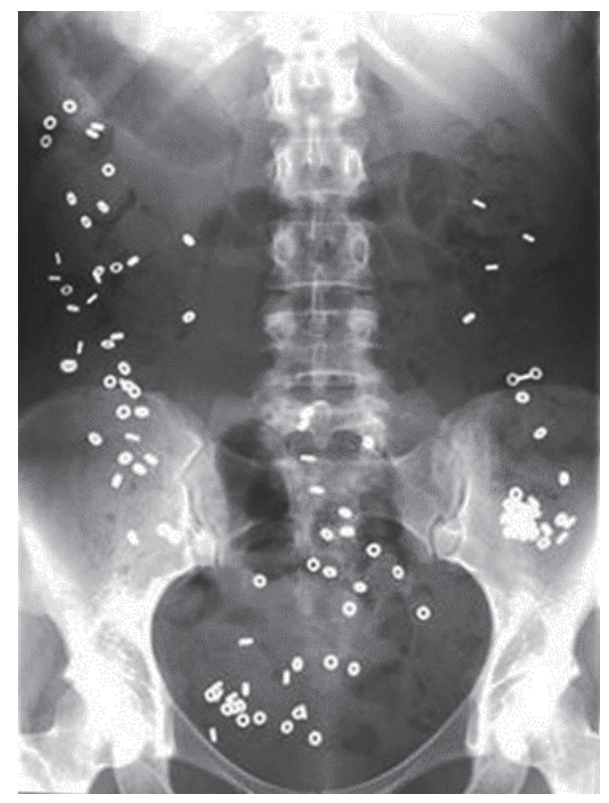

Abb. 21.3: $K T Z=$ Anzahl sichtbarer Markers $\times 2.4 \geq 144$ Stunden $=$ Slow-TransitObstipation.

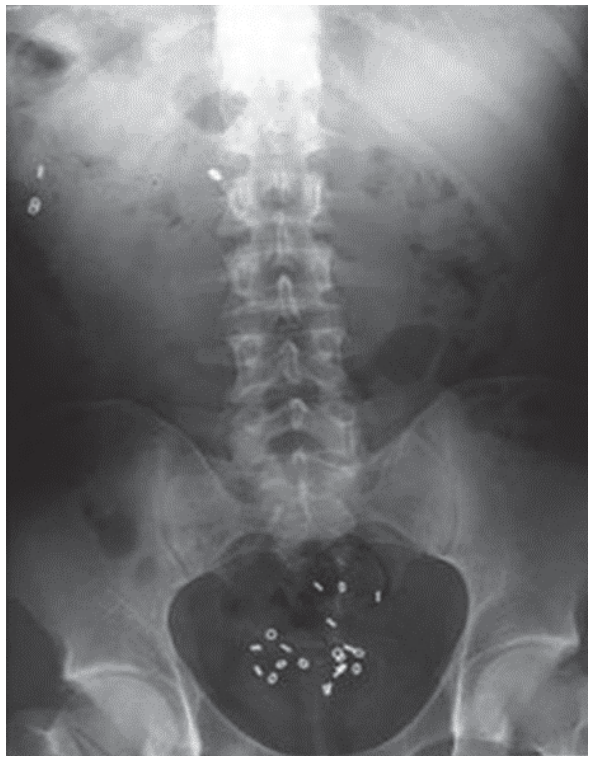

Abb. 21.4: $\mathrm{KTZ}=22 \times 2,4=53$ Stunden, normale Kolontransitzeit, einige Marker am Beckenboden, deshalb Verdacht auf OutletObstipation.

\subsubsection{Defäkographie}

In dieser Untersuchung wird der komplexe Entleerungsvorgang visualisiert und weitere Pathologien können ausgeschlossen werden. Insbesondere die Enterozele kann 
nur durch dieses Verfahren nachgewiesen werden. Die Defäkographie ist somit die wichtigste Untersuchung für die Darstellung des Entleerungsprozesses.

Sie kann mittels Durchleuchtung (Barium), Szintigrafie (Technetium-99m) oder im MRT durchgeführt werden. Die Szintigrafie ist im klinischen Alltag nicht relevant. Die konventionell-radiologische Untersuchung (Durchleuchtung) hat den Vorteil, dass sie im Sitzen, also in physiologischer Position, erfolgen kann. Zudem ist sie ein gutes Verfahren, wenn das MRI kontraindiziert ist, wie beispielsweise bei Schrittmacherträgern. Nachteilig ist die hohe Strahlenbelastung. Diese entfällt bei der MRT-Untersuchung, weshalb jene heutzutage am geläufigsten ist. Von Vorteil ist ein offenes MRT, da hier wie bei der Durchleuchtung durch die sitzende Position eine physiologische Situation geschaffen wird.

Bei der Beurteilung achte man auf den anorektalen Winkel, einen möglichen Beckenbodendeszensus, Rekto-, Entero- oder Zystozelen, Intussuszeptionen und Rektumprolapse.

Alle Untersuchungsmethoden zeigen ein dynamisches Bild der Defäkation, was dem behandelnden Arzt unmittelbare Rückschlüsse auf die Funktion des Darms und des Beckenbodens erlaubt.

\subsubsection{Anomanometrie}

Bis zu $75 \%$ der Patienten mit Obstipation zeigen in der Anomanometrie pathologische Resultate. Mit dieser Untersuchung gelingt die Darstellung von Dyssynergien des Beckenbodens oder auch die Diagnosestellung eines Morbus Hirschsprung.

Die High-Resolution-Anomanometrie (HRAM) ist ein relativ neues Verfahren, bei dem über einen Katheter mit mehreren Messpunkten Drücke vom Rektum bis zum anokutanen Übergang dokumentiert werden. Durch den Einsatz zusätzlicher Ballons können der Inhibitionsreflex oder Schwellenwerte für den Stuhldrang, Rektumkapazität usw. gemessen werden.

\subsubsection{Koloskopie und Biopsie}

Die Indikationsstellung für diese Untersuchung sollte grundsätzlich grosszügig erfolgen und ist als zwingend notwendig zu betrachten bei Patienten über 50 Jahren oder Vorhandensein von "Red flags”, d. h. Hinweisen auf eine mögliche Tumorerkrankung (veränderte Stuhlgewohnheiten, Blutabgang ab ano, B-Symptomatik). Auch wenn die konservative Therapie versagt und mit dem Patienten eine Operation diskutiert wird, sollte vorgängig eine endoskopische Abklärung des Kolons erfolgen. Für eine morphologische Beurteilung der Kolonwand ist ein Vollwandresektat nötig, was koloskopisch nur eingeschränkt und nur im Rektum möglich ist. Um eine aussagekräftige 
Biopsie zu erhalten, ist in der Regel eine transanale chirurgische Vollwandresektion im Rektum zu bevorzugen.

\subsubsection{Ballonexpulsionstest}

Er objektiviert, ab welchen Volumina der Patient subjektiv Stuhldrang verspürt und ob ein etwaiger Anismus möglicherweise die Defäkation verhindert. Der Ballonexpulsionstest kann für sich allein oder im Rahmen der Anomanometrie durchgeführt werden.

\subsubsection{Sonstiges}

Zusätzlich gibt es noch „wireless motility capsules“: es handelt sich hierbei um unverdaubare Kapseln, die der Patient schluckt und die pH-Level sowie selektiv verschiedene Transitzeiten des Magens, Dünndarms und Dickdarms messen. Diese Untersuchung belastet den Patienten nicht aufgrund der kleinen Kapselgrösse. Somit kann eine Aussage über allfällige Motilitätsstörungen des gesamten Verdauungstraktes getroffen werden und die Methode ist vor allem vor subtotaler Kolektomie sinnvoll, um den langfristigen Verlauf nach Operation abzuschätzen.

Bei zusätzlichem Verdacht auf eine Dünndarmmotilitätsstörung ist eine MRI Sellink eine empfohlene Untersuchung, um Stenosen oder Briden ausschliessen zu können.

\subsubsection{Therapie}

Oft stellt sich die Behandlung chronisch obstipierten Patienten als langwierig und anspruchsvoll heraus. Basis für jede Therapieform ist die suffiziente Compliance neben ausreichenden kognitiven Fähigkeiten des Patienten. Als Priorität bzw. Therapieziel müssen die Linderung der Symptome und eine Verbesserung der Lebensqualität angesehen werden. V. a. bei komplexeren Fällen lohnt sich eine interdisziplinäre Zusammenarbeit in Form eines Beckenboden-Boards, bei dem Chirurgen, Gastroenterologen, Urologen, Gynäkologen, Neurologen und Physiotherapeuten involviert sind.

Die Abklärungsschritte und die Therapie sollten sich an einem Stufenschema orientieren, wie es Abb. 21.5 und 21.6 zeigen. 


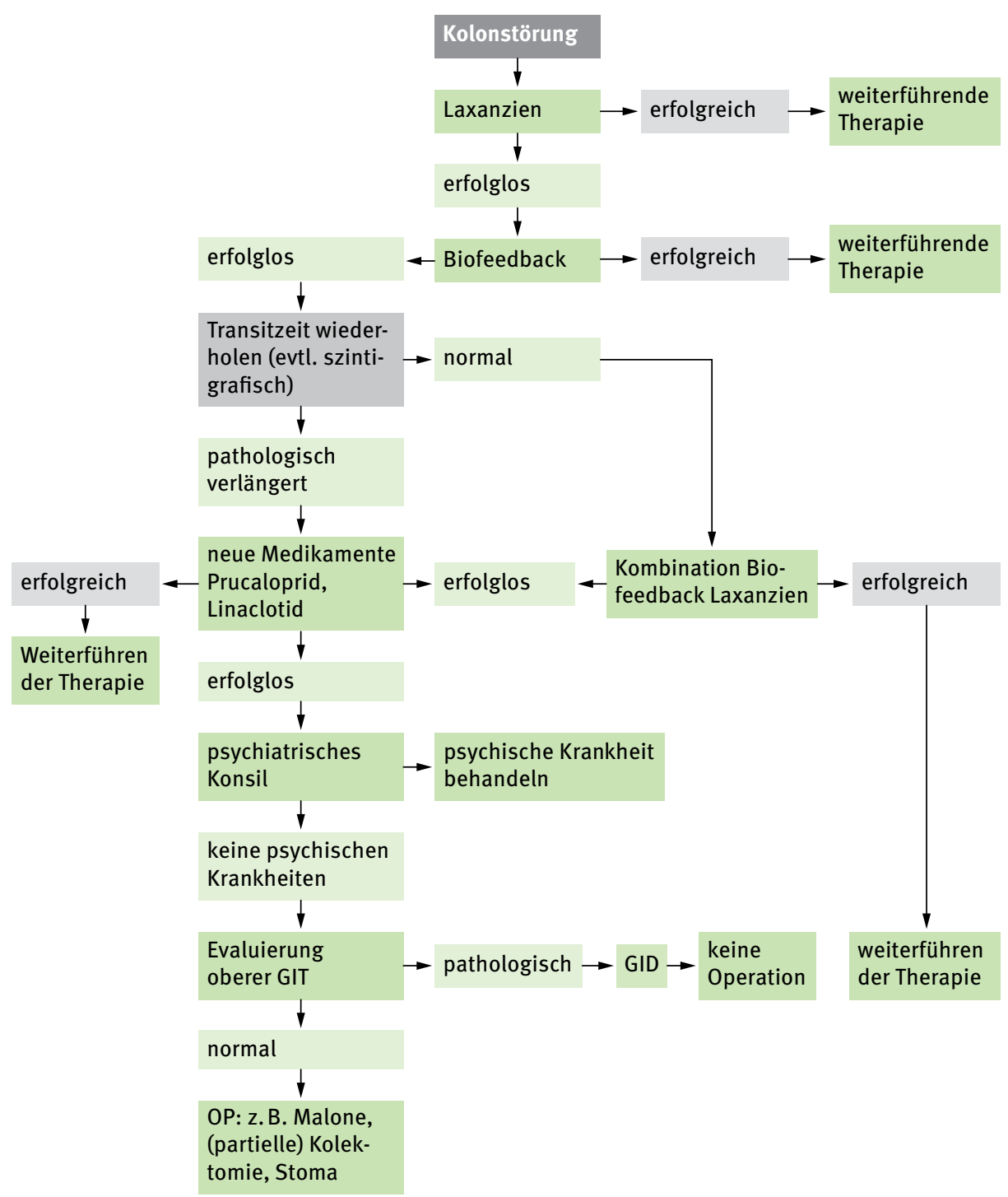

Abb. 21.5: Algorithmus zur Diagnostik und Therapie der Kolontransportstörung. 


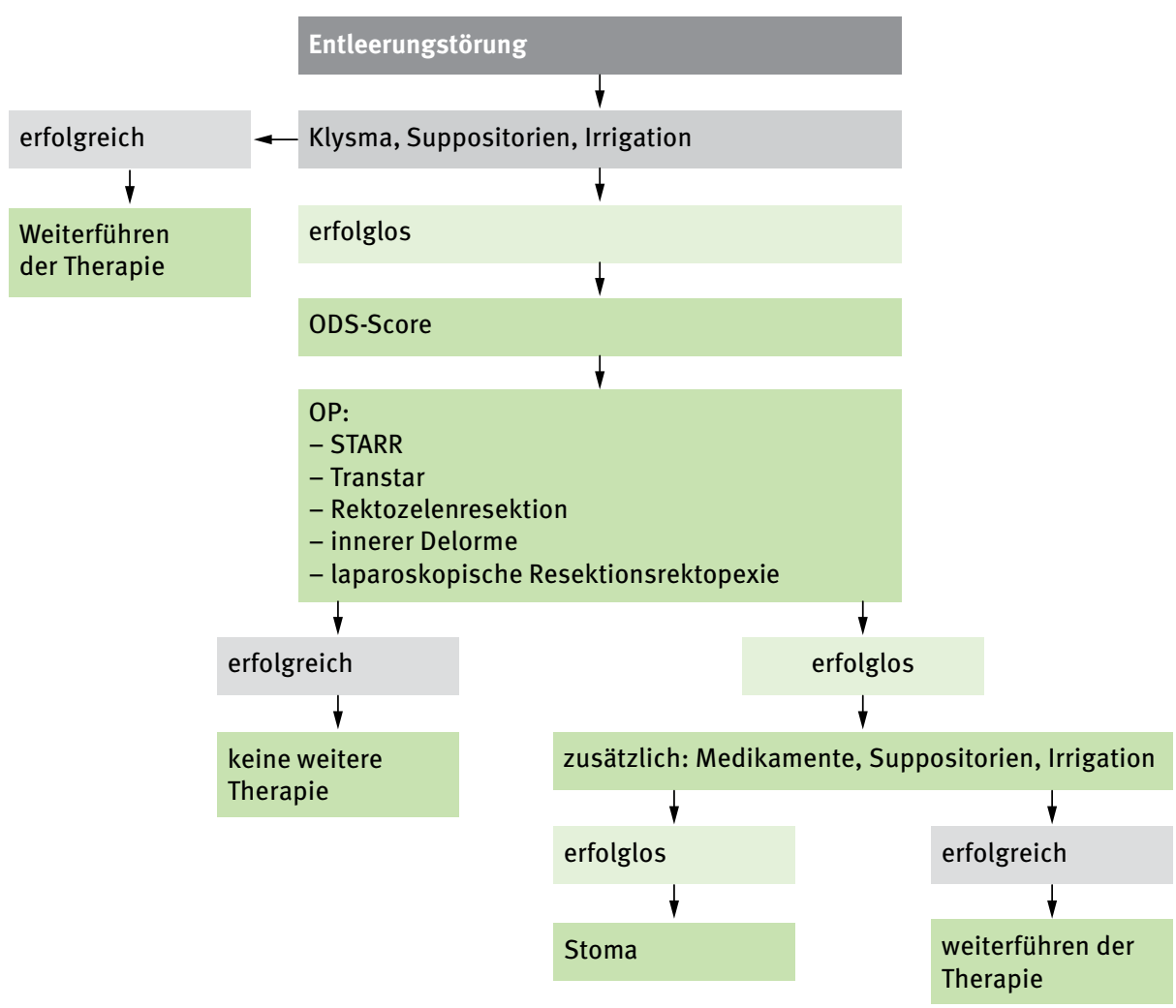

Abb.21.6: Algorithmus zur Diagnostik und Therapie der Entleerungsstörung.

\subsubsection{Konservative Therapie}

Vor dem Beginn einer medikamentösen Therapie sollte der Patient in einem ersten Schritt Allgemeinmaßnahmen ergreifen bzw. Lebensstilveränderungen durchführen. Diese beinhalten neben der körperlichen Betätigung eine ausreichende Trinkmenge sowie genügend ballaststoffreiche Kost. Sollte dies nicht ausreichen, wirken sich Quellmittel wie Flohsamenschalenpulver günstig aus. In einem nächsten Schritt kommen stimulierende oder osmotische wirksame Laxanzien zum Einsatz. Es kann nicht genug betont werden, wie wichtig eine gute Aufklärung des Patienten ist: Ziel ist eine regelmässige Stuhlfrequenz, jedoch muss der Stuhlgang nicht täglich erfolgen. Unter der Voraussetzung unauffälliger Basisuntersuchungen, fehlender Schmerzen und fehlender B-Symptomatik kann zunächst eine konservative Therapie eingeleitet und auf weiterführende Diagnostik verzichtet werden.

Neben der medikamentösen Therapie beinhaltet die konservative Therapie auch das Beckenbodentraining. Dieses hilft nicht nur Patienten mit Outlet-Obstipation: bei Patienten mit Beckenbodendyssynergie hat sich das Biofeedback bewährt, da sie 
durch die direkte Rückmeldung über die Muskelfunktion eine physiologische Defäkation (wieder)erlernen. Wenn der obstipierte Patient unter einer zu grossen Rektumkapazität oder rektaler Hyposensibilität leidet, führen Sensibilisierungsübungen mit verschiedenen Ballonvolumina dazu, dass er die Ampullenfüllung wieder spürt und die geforderten Reflexe auslösen kann.

Für die Slow-Transit-Obstipation ist ein gutes, wenig invasives Verfahren die transanale rektale Irrigation. Es stehen verschieden Produkte zur Verfügung, die es dem Patienten erlauben, dies selbständig zuhause vorzunehmen. Falls diese Therapie erfolgreich ist, aber für den Patienten mühsam, kann eine anterograde Kolonspülung über eine Kolonfistel empfohlen werden (Malone-Procedure mit Appendixfistel).

\section{Sakralnervenmodulation}

Das Verfahren der Sakralnervenmodulation (SNM) oder auch -stimulation ist etabliert in der Therapie der Urin- und Stuhlinkontinenz. Die SNM hat in der Inkontinenzbehandlung die besseren Ergebnisse, sie kann jedoch auch in der Behandlung pelviner Schmerzen oder der Slow-Transit-Obstipation eingesetzt werden.

Einige Studien liefern Hinweise, dass die SNM auch langfristig erfolgreich sein kann, so in $73 \%$ der Fälle obstipierter Patienten. Diese Ergebnisse wurden allerdings durch neuere randomisiert-kontrollierte Studien in Frage gestellt, in denen sich anhaltende Verbesserungen nur bei 11-47\% zeigten. Bei der Therapie der Slow-Transit-Obstipation schnitt in diesen Studien die SNM nicht besser als Placebo ab. Da es sich aber um ein minimal invasives Verfahren handelt mit geringer Morbidität und in einem Testsetting 3-4 Wochen erproben lässt, kann es vor einem resezierenden Verfahren empfohlen werden.

\section{Periphere Tibialisnervenstimulation (PNTS)}

Die periphere Nervus tibialis Stimulation, ebenfalls ein Verfahren, das bei Blasenentleerungsstörungen erfolgreich angewandt wird, hat in wenigen Studien eine Wirkung bei der Slow-Transit-Obstipation ergeben. Zum Beispiel hat Collins et al. 2012 eine Tendenz zu einer verbesserten Stuhlentleerung und Lebensqualität bei 18 Patienten mit Slow-Transit-Obstipation aufzeigen können. Leider konnten diese guten Resultate im University College London 2017 an 30 Patienten nicht wiederholt werden. Der Stellenwert der PNTS bei der chronischen Obstipation ist also noch unklar.

\subsubsection{Operative Therapie}

Bevor ein operatives Verfahren auch nur in Erwägung gezogen wird, muss die Ursache der Obstipation evaluiert und die konservative Therapie voll ausgeschöpft worden sein. Erst nach frustranen konservativen Maßnahmen kommt die Operation als Therapieoption in Frage. Abhängig von der Ätiologie der Obstipation gibt es Eingriffe 
zur Behandlung der Slow-Transit-Obstipation und solche zur Therapie von Outlet-Obstipationen (vgl. Kapitel 13 Stuhlentleerungsstörungen, Band 1). Allen Operationsverfahren gemeinsam ist die Notwendigkeit einer engen Patientenführung. Dazu gehört die Aufklärung, dass der Patient von der Obstipation möglicherweise postoperativ nicht komplett geheilt ist und dass auch eine (passagere) Verschlechterung möglich ist. Zudem kann der Patient postoperativ aufgrund einer langjährigen Überdehnung des Beckenbodens eine Inkontinenz entwickeln.

\section{Slow-Transit-Obstipation}

Das Stellen der korrekten Operationsindikation ist schwierig und sollte koloproktologisch erfahrenen Ärzten vorbehalten sein. Eine operative Maßnahme darf nur nach ausführlicher Anamnese und Diagnostik ergriffen werden. Voraussetzung ist, dass sämtliche konservative Möglichkeiten ausgeschöpft und über Jahre hinweg vom Patienten konsequent durchgeführt worden sind. Da Motilitätsstörungen mitunter den gesamten Verdauungstrakt betreffen, klagen Patienten gelegentlich nach einer Kolonresektion weiterhin über Symptome wie beispielsweise ein Völlegefühl. Auch hier wiederum gilt es, den Patienten im Vorfeld ausführlich zu informieren, um überhöhten Erwartungen an das postoperative Ergebnis entgegenzuwirken.

Die zwei wichtigsten chirurgischen Therapieoptionen bei der Slow-Transit-Obstipation sind die totale Kolektomie mit ileorektaler Anastomose und die subtotale Kolektomie. Eine segmentale Kolektomie kann auch vorgenommen werden, ist aber mit einer viel geringeren Erfolgsrate versehen und wenn überhaupt nur in selektionierten Fällen sinnvoll.

\section{Kolektomie mit ileorektaler Anastomose}

Die postoperativen Resultate nach Kolektomie durch erfahrene Operateure sind überzeugend, obgleich der Eingriff selbstredend als Ultima Ratio anzusehen ist (Abb. 21.7). Wichtig ist im Vorfeld die differentialdiagnostische Unterscheidung zwischen Patienten mit Slow-Transit-Obstipation von denjenigen mit Normal-Transit-Obstipation oder Patienten mit Reizdarmsyndrom, da nur das erstgenannte Patientenkollektiv von einer Kolonresektion profitiert. Nahezu $90 \%$ der operierten Patienten benötigen keinerlei Laxanzien mehr und beschreiben eine signifikante Regredienz der Beschwerden mit verbesserter Lebensqualität (Tab. 21.5). Die Indikation für einen resezierenden Eingriff ist zurückhaltend zu stellen bei Patienten mit Schmerzen oder ausgeprägten Flatulenzen, da hier häufig ein generelles Motilitätsproblem vorliegt. Ggf. kann probatorisch die Anlage einer Ileostomie erfolgen. Im Fall einer Persistenz der Symptomatik ist die Kolektomie nicht erfolgversprechend und entsprechend kontraindiziert.

Die totale Kolektomie mit ileorektaler Anastomose wird bevorzugt heute laparoskopisch oder laparoskopisch handassistiert durchgeführt und weist eine geringe Morbidität und Mortalität (0-15\%) auf, da es sich in der Regel um junge und sonst 


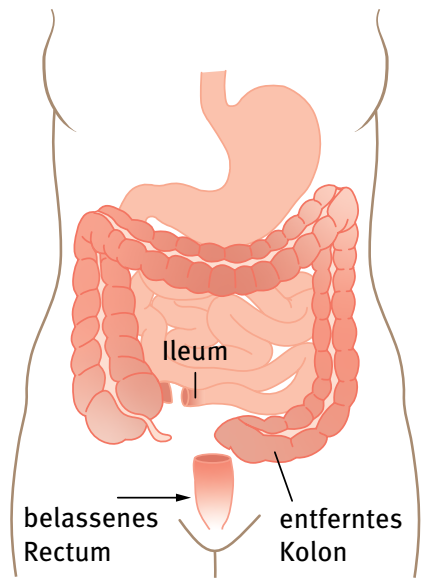

(a)

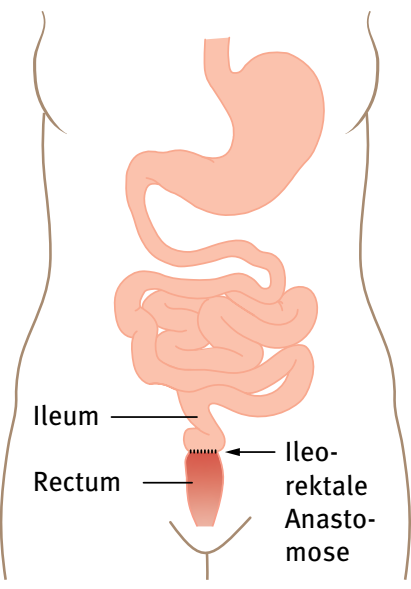

(b)
Abb. 21.7: Kolektomie mit ileorektaler Anastomose.

gesunde Patienten handelt. Die häufigste postoperative Komplikation ist eine Dünndarmpassagestörung, ausgelöst durch Briden oder eine neurologische Fehlfunktion des Plexus myentericus. Unspezifische Bauchschmerzen sind selten, aber eine schwierig zu behandelnde Komplikation im Langzeitverlauf nach Kolektomie. Bei hohen Stuhlfrequenzen müssen die Patienten ggf. lebenslang betreffend Gallensalzverlustsyndrom und Vitamin B12 Mangelerscheinungen monitorisiert werden.

Tab. 21.5: Totale Kolektomie und ileorektale Anastomose.

\begin{tabular}{llll}
\hline Autor & Evaluierte Patienten & Operierte Patienten & Erfolgsrate (\%) \\
\hline Pemberton et al., 1991 & 277 & 38 & 100 \\
\hline Wexner et al., 1991 & 163 & 16 & 94 \\
\hline Sunderland et al., 1992 & 228 & 18 & 88 \\
\hline Mehendra et al., 1994 & 19 & 9 & 88 \\
\hline Redmond et al., 1995 & 37 & 37 & 90 \\
\hline Pluta et al., 1996 & 180 & 24 & 90 \\
\hline Nyam et al., 1997 & 1009 & 74 & 97 \\
\hline Fitz et al., 2003 & - & 112 & 93 \\
\hline Feng et al., 2008 & - & 45 & 93 \\
\hline Mollen et al., 2011 & 439 & 21 & 52 \\
\hline Sohn et al., 2011 & - & 37 & 82 \\
\hline Vergara-Fernandez., 2014 & 710 & 80 & 88 \\
\hline
\end{tabular}




\section{Subtotale Kolektomie}

Die subtotale Kolektomie kann eine gute Alternative zur Kolektomie sein. Die Resektion beginnt $10 \mathrm{~cm}$ distal der ileozökalen Verbindung und endet im oberen Drittel des Rektums (Abb. 21.8). Der Erhalt des terminalen Ileums, der ileozökalen Klappe und des Zökums erlaubt eine Reabsorbtion von Elektrolyten, Gallensalzen, Vitamin B12 und etwa 2 Liter Wasser pro Tag. Betreffend Effektivität und Sicherheit scheint dieses Verfahren etwa wie die totale Kolektomie zu sein, mit einer etwas geringeren Rate von Durchfällen und Inkontinenzepisoden. In einer vergleichenden Studie von 2008 schnitt die totale Kolektomie mit ileorektaler Anastomose aber betreffend Stuhlfrequenz, Verbrauch von Laxanzien und Lebensqualität deutlich besser ab. Eine klare Empfehlung für eine der beiden resezierenden Verfahren kann zum heutigen Zeitpunkt nicht abgegeben werden.

\section{Segmentresektion des Kolons}

Obwohl die segmentale Resektion eine attraktive Option sein könnte, besteht die Schwierigkeit, das zu resezierende Kolonsegment anhand von Transitstudien zu definieren. Oder anders ausgedrückt, stellt sich die Frage, welcher Teil des Kolons belassen werden kann, ohne dass postoperativ Obstipationsbeschwerden persistieren. Ist das der Fall, ist eine weitere Resektion nötig, wie es auch nach einer subtotalen Kolektomie vorkommen kann.
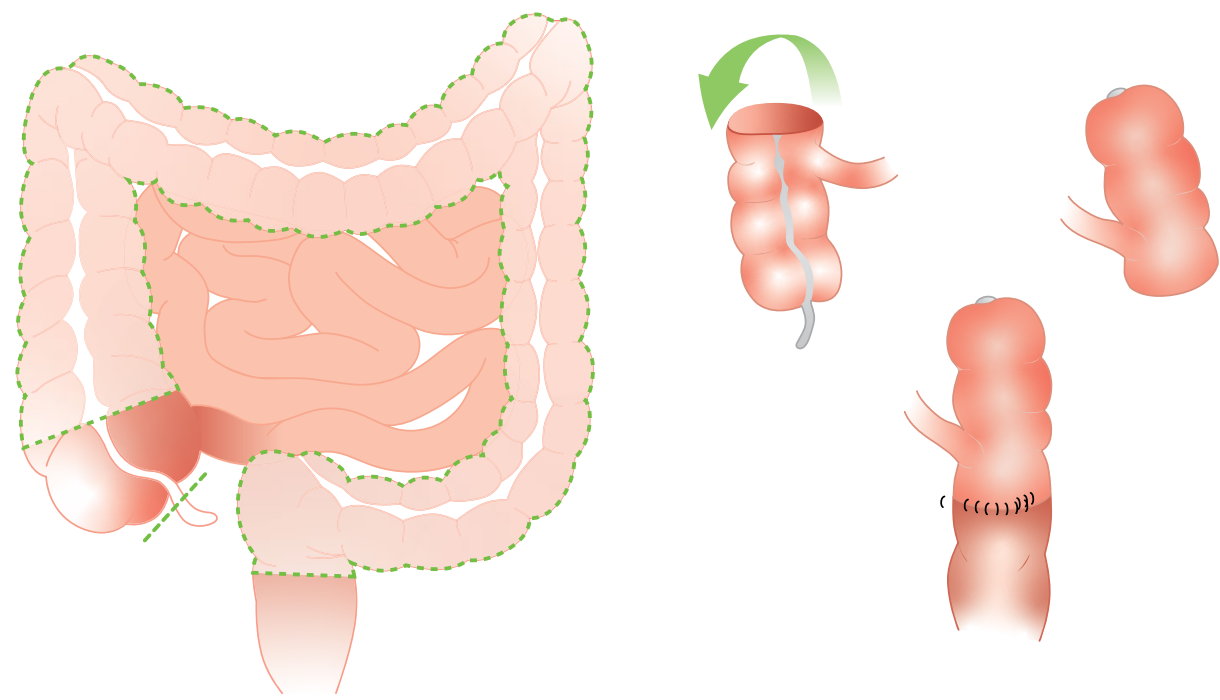

Abb. 21.8: Subtotale Kolektomie mit Zökorektostomie. Das Zökum wird 180 Grad im Gegenuhrzeigersinn gedreht, so dass das terminale Ileum von rechts einmündet. 


\section{Weiterführende Literatur}

Andresen V, Enck P, Frieling T, et al. S2k-Leitlinie Chronische Obstipation: Definition, Pathophysiologie, Diagnostik und Therapie. Z Gastroenterol. 2013;51:651-672.

Arebi N, Kalli T, Howson W, et al. Systematic review of abdominal surgery for chronic idiopathic constipation. Colorectal Dis. 2011;3:1335-1343.

Baeten CGMI, Beglinger C, Degen L, et al. Proktologie. In: Siewert JR, Schumpelick V, Rothmund M, Hrsg. Praxis der Viszeralchirurgie Gastroenterologische Chirurgie. 3. Aufl. Berlin, Heidelberg: Springer; 2011, 585-644.

Bharucha AE, Pemberton JH, Locke GR. American gastroenterological association technical review on constipation. Gastro enterology. 2013;144:218-238.

Buchmann P. Constipation. In: Herold A, Lehur P-A, Matzel KE, O'Connell PR, eds. Coloproctology. Berlin, Heidelberg: Springer; 2008: 93-104.

Collins B, Norton C, Maeda Y. Percutaneous tibial nerve stimulation for slow transit constipation: a pilot study. Colorectal Dis. 2012;14(4):e165-170.

De Marco P, Militello G, Tutino R, et al. The management of slow transit constipation in the laparoscopic era. G Chir. 2018;34:297-302.

Dinning PG, Hunt L, Patton V, et al. Treatment efficacy of sacral nerve stimulation in slow transit constipation: a two-phase, double-blind randomized controlled crossover study. Am J Gastroenterol. 2015;110:733-740.

Feng Y, Jianjiang L. Functional outcomes of two types of subtotal colectomy for slow-transit constipation: ileosigmoidal anastomosis and cecorectal anastomosis. Am J Surg. 2008;195(1):73-77.

Graf W, Sonesson AC, Lindberg B, et al. Results after sacral nerve stimulation for chronic constipation. Neurogastroenter- ol Motil. 2015;27:734-739.

Frühauf H, Fox MR. Obstipation. Gastroenterologe. 2008;3:488-496.

Herold A. Diagnostik und chirurgische Therapie der Chronischen Obstipation. Viszeralchirurgie. 2001;36:196-202.

Kamm MA, Dudding TC, Melenhorst J, et al. Sacral nerve stimulation for intractable constipation. Gut. 2010;59:333-340.

Kumar L, Liwanag J, Athanasakos E, et al. Effectiveness of percutaneous tibial nerve stimulation in managing refractory constipation. Colorectal Dis. 2017;19(1):45-49.

Mugie SM, Benninga MA, Di Lorenzo C. Epidemiology of constipation in children and adults: a systematic review. Best Pract Res Clin Gastroenterol. 2011;25:3-18.

Peppas G, Alexiou VG, Mourtzoukou E, et al. Epidemiology of constipation in Europe and Oceania: a systematic review. BMC Gastroenterol. 2008;8:5.

Ratto C, Ganio E, Naldini G. Long-term results following sacral nerve stimulation for chronic constipation. Colorectal Dis. 2015;17:320-328.

Sailer M, Aigner F, Hetzer F, Hrsg. Expertise Koloproktologie. 1. Aufl. Stuttgart: Thieme; 2016.

Senapati A, Gray RG, Middleton LJ, et al. PROSPER: a random- ised comparison of surgical treatments for rectal prolapse. Colorectal Dis. 2013;15:858-868.

Soares ASGF, Barbosa LER. Colonic Inertia: approach and treatment. J Coloproctol. 2017;37:63-71.

Tack J, Müller-Lissner S, Stanghellini V, et al. Diagnosis and treatment of chronic constipation - a European perspective. Neurogastroenterol Motil. 2011;23:697-710.

Thaha MA, Abukar AA, Thin NN, Ramsanahie A, Knowles CH. Sacral nerve stimulation for faecal incontinence and constipation in adults. Cochrane Database Syst Rev. 2015;24;(8): Review.

Vergara-Fernandez O, Mejía-Ovalle R, Salgado-Nesme N, et al. Functional outcomes and quality of life in patients treated with laparoscopic total colectomy for colonic inertia. Surg Today. 2014;44(1):34-38.

Zerbib F, Siproudhis L, Lehur PA, et al. Randomized clinical trial of sacral nerve stimulation for refractory constipation. Br J Surg. 2017;104:205-213. 
